\title{
AV Block Second Degree
}

National Cancer Institute

\section{Source}

National Cancer Institute. AV Block Second Degree. NCI Thesaurus. Code C111119.

Intermittent failure of atrial electrical impulse conduction to the ventricles. 